\title{
Molecular characterisation of sweet cherry (Prunus avium L.) genotypes using peach [Prunus persica (L.) Batsch] SSR sequences
}

\author{
A Wünsch ${ }^{1}$ and JI Hormaza ${ }^{1,2}$ \\ ${ }^{1}$ Unidad de Fruticultura, Servicio de Investigación Agroalimentaria, Campus de Aula Dei, Zaragoza, Spain
}

A total of 76 sweet cherry genotypes were screened with 34 microsatellite primer pairs previously developed in peach. Amplification of SSR loci was obtained for 24 of the microsatellite primer pairs, and 14 of them produced polymorphic amplification patterns. On the basis of polymorphism and quality of amplification, a set of nine primer pairs and the resulting 27 informative alleles were used to identify 72 genotype profiles. Of these, 68 correspond to unique cultivar genotypes, and the remaining four correspond to three cultivars that could not be differentiated from the two original genotypes of which they are mutants, and two very closely related cultivars. The mean number of alleles per locus was
3.7 while the mean heterozygosity over the nine polymorphic loci averaged 0.49 . The results demonstrate the usefulness of cross-species transferability of microsatellite sequences allowing the discrimination of different genotypes of a fruit tree species with sequences developed in other species of the same genus. UPGMA cluster analysis of the similarity data divided the ancient genotypes studied into two fairly well-defined groups that reflect their geographic origin, one with genotypes originating in southern Europe and the other with the genotypes from northern Europe and North America.

Heredity (2002) 89, 56-63. doi:10.1038/sj.hdy.6800101

Keywords: cultivar identification; fingerprinting; SSR transferability; molecular markers; microsatellites

\section{Introduction}

Sweet cherry (Prunus avium L., Rosaceae, $2 n=16$ ) is cultivated in temperate regions of the world for its edible fruit. Cherries are thought to have originated around the Caspian and Black Seas and currently they are found across mainland Europe and in western Asian areas (Webster, 1996). Sweet cherry cultivation spread through Europe in times of the Roman Empire, although they were not planted extensively until the 16th century (Watkins, 1976). The spread of sweet cherry cultivation across western Europe was probably based on domestication of wild individuals that were well adapted to each area of cultivation. A limited number of these cultivars were taken, by European settlers, to North America and spread throughout the continent. However, since sweet cherries were not commercially exploited until a century ago (Hedrick et al, 1915), most sweet cherry cultivars grown today are only a few generations from these landraces (Iezzoni et al, 1990).

Current breeding objectives have triggered a constant release of genetically improved genotypes (Iezzoni et al,

Correspondence: A Wünsch, Unidad de Fruticultura, Servicio de Investigación Agroalimentaria, Campus de Aula Dei, Apdo. 727, 50080 Zaragoza, Spain. E-mail: awunsch@aragob.es

${ }^{2}$ Current address: Estación Experimental la Mayora, CSIC, 29750 Algarrobo-Costa, Málaga, Spain. E-mail: ihormaza@eelm.csic.es Received 5 June 2001; accepted 14 February 2002
1990; Brown et al, 1996). Precise identification of the existing sweet cherry cultivars is essential for sweet cherry orchard establishment, efficient germplasm collection management, and selection of genotypes in cherry breeding programmes. Moreover, efficient characterisation methods are needed to assess the precious germplasm still present in eastern Europe and to study variability in wild stands scattered through Eurasia.

Cultivar identification has traditionally been based on morphological traits although DNA markers allow a more precise identification of plant genotypes and are of great interest for the characterisation of fruit trees. However, little work has been dedicated to the identification of sweet cherry cultivars. Thus, Granger et al (1993) and Beaver et al (1995) used isozymes to identify unique genotype profiles from sweet cherry cultivars whilst Gerlach and Stösser (1997) differentiated sweet cherry cultivars using RAPD markers.

Microsatellite markers (SSR; Litt and Luly, 1989; Tautz, 1989) are becoming the markers of choice for fingerprinting purposes due to their high polymorphism and reproducibility (Gupta and Varshney, 2000; Wünsch and Hormaza, in press). Among temperate fruit tree species, most microsatellite flanking sequences have been cloned and sequenced in peach (Prunus persica (L.) Batsch; Cipriani et al, 1999; Sosinski et al, 2000; Testolin et al, 2000; Aranzana et al, 2002) and apple (Malus $\times$ domestica Borkh.; Guilford et al , 1997; Gianfranceschi et al, 1998; Hokanson et al, 1998), where they have been used in genotype identification and the determination of genetic relatedness. One drawback of microsatellites is the 
difficulty of their initial isolation. However, once the primer sequences have been identified, SSR markers can be easily applied and the flanking sequences are frequently conserved among species and genera (Downey and Iezzoni, 2000; Hormaza, 2002; Yamamoto et al, 2001). However, studies of cross-species transferability in temperate fruit tree species have included limited numbers of genotypes and studies at a larger scale are required to validate the widespread use of the currently available SSR sequences. Thus, in this work, we have tested 34 microsatellite primer pairs developed in peach to characterise and study the genetic similarities among 76 sweet cherry genotypes, including old cultivars obtained from diverse geographical regions, and new genotypes released from different cherry breeding programmes.

\section{Materials and methods}

\section{Plant material and genomic DNA extraction}

Genomic DNA was extracted from 76 sweet cherry genotypes (Table 1) maintained at the SIA-DGA experimental orchards located at the Campus de Aula Dei in Zaragoza, Spain, using a modified version (Hormaza, 1999) of the DNA extraction protocol described by Doyle and Doyle (1987). Since low quality DNA was extracted from old leaves and fresh leaves are only available in spring, genomic DNA was extracted from eight to 10 winter buds collected during November and December. DNA from fresh leaves collected in spring was compared with that obtained from winter buds for 10 genotypes. Extracted DNA was quantified spectrophotometrically and diluted to $10 \mathrm{ng} / \mu \mathrm{l}$ before PCR amplification.

\section{PCR amplification and product electrophoresis}

Extracted genomic DNA was PCR-amplified using 34 pairs of microsatellite primers developed in peach (Tables 2 and 3). PCR reactions were performed in $20 \mu \mathrm{l}$ volumes containing $20 \mathrm{mM}$ Tris- $\mathrm{HCl}, \mathrm{pH} 8.4,50 \mathrm{mM} \mathrm{KCl}$, $4 \mathrm{mM} \mathrm{MgCl}_{2}, 0.1 \mathrm{mM}$ each dNTP, $0.2 \mu \mathrm{M}$ each primer, $40 \mathrm{ng}$ genomic DNA, 0.45 units Taq polymerase (Life Technologies, Rockville, MD, USA) and a drop of mineral oil. Reactions were carried out on a PTC-100 (MJ Research) thermocycler using the following temperature profile: an initial step of $2 \mathrm{~min}$ at $94^{\circ} \mathrm{C}, 35$ cycles of 45 sec at $94^{\circ} \mathrm{C}, 45 \mathrm{sec}$ at $57^{\circ} \mathrm{C}$ and $1 \mathrm{~min}$ at $72^{\circ} \mathrm{C}$, and a final step of $5 \mathrm{~min}$ at $72^{\circ} \mathrm{C}$. For primers pchcms 1 and pchcms 3 , the annealing temperature was lowered to $40^{\circ} \mathrm{C}$. PCR products were separated by electrophoresis using $3 \%$ 'Metaphor' agarose gels (FMC Bioproducts) in $1 \times \mathrm{TBE}$ buffer at $5 \mathrm{~V} / \mathrm{cm}$, stained with ethidium bromide and visualised under UV light. Band scoring was carried out using a standard $10 \mathrm{bp}$ DNA ladder (Life Technologies). To confirm the results, microsatellite products that revealed differences smaller than $5 \mathrm{bp}$ in agarose gels, were also separated using $10 \%$ polyacrylamide gel electrophoresis in $1 \times \mathrm{TBE}$ buffer at a constant current of $40 \mathrm{~mA}$, stained with ethidium bromide and visualised under UV light.

\section{Data analysis}

Heterozygosity was calculated as the number of heterozygous individuals over the total number of individuals analysed for each locus and averaged over the polymorphic loci. Genetic relationships among the genotypes

were calculated using UPGMA cluster analysis of the similarity matrix obtained from the proportion of shared fragments (Nei and Li, 1979). Cophenetic coefficients were computed for the dendrograms after the construction of a cophenetic matrix. All those analyses were computed with the programme NTSYS-pc 2.02 (Exeter Software, Stauket, New York, NY, USA). The robustness of the dendrogram obtained from UPGMA cluster analysis was assessed with bootstrap analysis running 2000 iterations using the WinBoot program (Yap and Nelson, 1996).

\section{Results}

Repeatable amplification was obtained for 24 (70\%) of the 34 microsatellite primer pairs tested, 13 (38\%) were polymorphic and nine of these (Table 2) were selected for the identification of the 76 sweet cherry genotypes. As expected, no differences were recorded between the amplification patterns among the samples taken from fresh leaf tissue in spring and dormant winter buds. Nondenaturing polyacrylamide gel electrophoresis confirmed the results using 'Metaphor' agarose gel electrophoresis but revealed no additional bands. In fact, polyacrylamide electrophoresis showed greater numbers of artefact and stutter bands, making identification of microsatellite alleles more difficult than with agarose gel electrophoresis.

Out of the 24 primer pairs that amplified either polyor monomorphic loci, 11 (45\%) of them showed alleles in the same size range as those reported in peach for the same primer pairs (Tables 2 and 3). Four of the 13 polymorphic loci were discarded, because either size differences among bands were too small to be accurately scored (Pchcms1, UPD98-024, UPD98-410) or complex band patterns (UPD98-411) did not allow identification of unique loci. Three primer pairs (Pchms3, UPD98-409, UPD98-412) amplified alleles common to all genotypes analysed. The number of alleles per locus ranged from two to seven, ranging from $90 \mathrm{bp}$ to $290 \mathrm{bp}$. Heterozygosity ranged from 0.04 to 0.94 for loci UPD98-409 and UPD96-005 respectively, the mean heterozygosity being 0.49 . Heterozygosity for the 23 ancient and/or of unknown pedigree cultivars was 0.49 , whilst heterozygosity for the other 53 genotypes from more recent breeding programmes was 0.47 .

Genotypes showing a single amplified fragment were considered homozygous for that particular locus (Callen et al, 1993). Inheritance of microsatellites was checked for the genotypes with known pedigree and whose parental genotypes were present in our collection. In all cases, bands present in the progeny were always present in at least one of the parental genotypes. Rare alleles were amplified with primer pairs UPD98-409, UPD98-412, UPD97-402 and UDP98-022. All the sampled cherry genotypes present a common allele of $130 \mathrm{bp}$ with primer pair UPD98-409 and only 'Ambrunes', 'Pico Negro' and 'Pico Colorado' show a second band of $160 \mathrm{bp}$. The cultivars 'Marmotte', 'Hedelfinger' and 'Blanca de Provenza' share a distinct allele with primer pair UPD98-412. Generally, old cultivars, eg 'Burlat', 'Marmotte' or 'Hedelfinger', and several Spanish cultivars contributed most of the rare alleles.

The combination of nine selected primers and the resulting 27 informative markers allowed the unambigu- 
Table 1 Sweet cherry cultivars included in this study with the SSR genotypes obtained after amplification with nine SSR primer pairs (primer details in Table 2)

Origin* Pchgms1 Pchcms3 Pchcms5 UDP96-005 UDP98-409 UPD98-021 UPD98-022 UPD97-402 UPD98-412

\begin{tabular}{|c|c|c|c|c|c|c|c|c|c|c|}
\hline Ambrunes & $\mathrm{U}, \mathrm{s}$ & 140 & $180 / 160$ & 290 & $150 / 125$ & $160 / 130$ & $110 / 100$ & $110 / 90$ & 125 & 130 \\
\hline Arcina & $\mathrm{BP}, \mathrm{F}$ & 140 & 180 & 260 & $150 / 130$ & 130 & $110 / 100$ & $100 / 90$ & $140 / 125$ & 130 \\
\hline Belge & $\mathrm{U}, \mathrm{CE}$ & $190 / 140$ & 180 & 260 & $150 / 120$ & 130 & $110 / 100$ & $110 / 90$ & 130 & 130 \\
\hline Bing & $\mathrm{BP}, \mathrm{US}$ & $190 / 140$ & 180 & 260 & $150 / 120$ & 130 & $110 / 100$ & $110 / 90$ & 130 & 130 \\
\hline Blanca de Provenza & $\mathrm{U}$ & 140 & $180 / 160$ & $290 / 260$ & 120 & 130 & 100 & $105 / 90$ & $145 / 125$ & $130 / 100$ \\
\hline Brooks & BP, US & 140 & 180 & 290 & $150 / 120$ & 130 & $110 / 100$ & 90 & $135 / 125$ & 130 \\
\hline Burlat & $\mathrm{U}, \mathrm{F}$ & 140 & 180 & 290 & $150 / 130$ & 130 & $110 / 100$ & $100 / 90$ & $130 / 120$ & 130 \\
\hline Burlat C-1 & $\mathrm{BP}, \mathrm{I}$ & 140 & 180 & 290 & $150 / 130$ & 130 & $110 / 100$ & $100 / 90$ & $130 / 120$ & 130 \\
\hline Celeste (Sumpaca) & $\mathrm{BP}, \mathrm{C}$ & 140 & 180 & 290 & $150 / 130$ & 130 & $110 / 100$ & 100 & $135 / 125$ & 130 \\
\hline Chinook & BP, US & $190 / 140$ & 180 & 260 & $150 / 120$ & 130 & $110 / 100$ & 110 & $145 / 130$ & 130 \\
\hline Compact Stella & $\mathrm{BP}, \mathrm{C}$ & $190 / 140$ & 180 & $290 / 260$ & $150 / 120$ & 130 & $110 / 100$ & $110 / 100$ & $145 / 125$ & 130 \\
\hline Coralise (Gardel) & $\mathrm{BP}, \mathrm{F}$ & 140 & 180 & 260 & $150 / 130$ & 130 & $110 / 100$ & $100 / 90$ & $135 / 125$ & 130 \\
\hline Corum & BP, US & $190 / 140$ & 180 & $290 / 260$ & $150 / 120$ & 130 & $110 / 100$ & $110 / 100$ & $135 / 125$ & $140 / 130$ \\
\hline Cristalina (Sumnue) & $\mathrm{BP}, \mathrm{C}$ & $190 / 140$ & $180 / 160$ & 260 & $150 / 120$ & 130 & 110 & $110 / 100$ & $135 / 125$ & 130 \\
\hline Cristobalina & $\mathrm{U}, \mathrm{S}$ & $190 / 140$ & 180 & $290 / 260$ & $150 / 115$ & 130 & $110 / 100$ & $110 / 90$ & $135 / 125$ & 130 \\
\hline Duroni 3 & $\mathrm{U}, \mathrm{I}$ & 140 & 180 & $290 / 260$ & $150 / 115$ & 130 & $110 / 100$ & $100 / 90$ & 125 & 130 \\
\hline Earlise (Riverdel) & $\mathrm{BP}, \mathrm{F}$ & 140 & 180 & $290 / 260$ & $150 / 130$ & 130 & 100 & 90 & $130 / 120$ & 130 \\
\hline Earlystar & $\mathrm{BP}, \mathrm{I}$ & $190 / 140$ & 180 & 290 & $150 / 120$ & 130 & 100 & $100 / 90$ & $145 / 125$ & 130 \\
\hline Early Van Compact & $\mathrm{BP}, \mathrm{C}$ & $190 / 140$ & 180 & $290 / 260$ & $150 / 120$ & 130 & 110 & $100 / 90$ & $135 / 125$ & $130 / 120$ \\
\hline Ferrovia & U, I & 140 & 180 & $290 / 260$ & $150 / 120$ & 130 & 110 & 100 & $145 / 130$ & $140 / 130$ \\
\hline Garnet (Magar) & $\mathrm{BP}, \mathrm{US}$ & 140 & 180 & 260 & 120 & 130 & $110 / 100$ & $110 / 90$ & 130 & 130 \\
\hline Gil Peck & BP, US & $190 / 140$ & $180 / 160$ & 260 & $150 / 120$ & 130 & 110 & 110 & $145 / 130$ & 130 \\
\hline Giorgia & $\mathrm{BP}, \mathrm{I}$ & 140 & 180 & $290 / 260$ & $150 / 120$ & 130 & 110 & $100 / 90$ & 125 & $130 / 120$ \\
\hline Hartland & $\mathrm{BP}, \mathrm{US}$ & 140 & 180 & 260 & $150 / 120$ & 130 & 110 & $100 / 90$ & $140 / 130$ & 130 \\
\hline Hedelfinger & $\mathrm{U}, \mathrm{CE}$ & 140 & 180 & $290 / 260$ & $150 / 120$ & 130 & $110 / 100$ & $110 / 100$ & $135 / 125$ & $130 / 100$ \\
\hline Lambert & $\mathrm{BP}, \mathrm{US}$ & $190 / 140$ & $180 / 160$ & $290 / 260$ & $150 / 120$ & 130 & $110 / 100$ & 110 & 125 & 130 \\
\hline Lamida & BP, US & 140 & 180 & 260 & $150 / 120$ & 130 & 110 & 110 & $145 / 125$ & 130 \\
\hline Lapins & $\mathrm{BP}, \mathrm{C}$ & $190 / 140$ & 180 & 290 & $150 / 120$ & 130 & $110 / 100$ & $100 / 90$ & $135 / 125$ & 130 \\
\hline Larian & $\mathrm{BP}, \mathrm{US}$ & $190 / 140$ & 180 & 260 & $150 / 120$ & 130 & 110 & $110 / 100$ & $145 / 130$ & 130 \\
\hline Marmotte & $\mathrm{U}, \mathrm{F}$ & $190 / 140$ & 180 & $290 / 260$ & $150 / 120$ & 130 & $110 / 100$ & $110 / 100$ & $135 / 125$ & $130 / 100$ \\
\hline Marvin (Niram) & $\mathrm{BP}, \mathrm{US}$ & 140 & 180 & $290 / 260$ & 120 & 130 & $110 / 100$ & $100 / 90$ & $130 / 125$ & 130 \\
\hline Moreau & $\mathrm{U}, \mathrm{F}$ & 140 & 180 & $290 / 260$ & $150 / 115$ & 130 & 100 & $100 / 90$ & $145 / 125$ & 130 \\
\hline Napoleon & $\mathrm{U}, \mathrm{CE}$ & $190 / 140$ & 180 & 260 & $150 / 120$ & 130 & $110 / 100$ & $110 / 100$ & $135 / 125$ & 130 \\
\hline Newstar & $\mathrm{BP}, \mathrm{C}$ & 140 & 180 & $290 / 260$ & $150 / 120$ & 130 & $110 / 100$ & 100 & $135 / 125$ & $130 / 120$ \\
\hline Pico Colorado & $\mathrm{U}, \mathrm{S}$ & 140 & $180 / 160$ & $290 / 260$ & $150 / 130$ & $160 / 130$ & $110 / 100$ & 110 & $140 / 125$ & 130 \\
\hline Pico Negro & $\mathrm{U}, \mathrm{S}$ & 140 & $180 / 160$ & $290 / 260$ & $150 / 115$ & $160 / 130$ & 100 & $110 / 90$ & $140 / 120$ & 130 \\
\hline Precoce Bernard & $\mathrm{U}, \mathrm{F}$ & 140 & 180 & $290 / 260$ & $150 / 130$ & 130 & 100 & $100 / 90$ & $145 / 130$ & 130 \\
\hline Rainier & $\mathrm{BP}, \mathrm{US}$ & $190 / 140$ & 180 & $290 / 260$ & 120 & 130 & $110 / 100$ & 90 & 130 & 130 \\
\hline Ramon Oliva & $\mathrm{U}, \mathrm{F}$ & 140 & $180 / 160$ & 290 & $150 / 130$ & 130 & 100 & 90 & 120 & 130 \\
\hline Reverchon & U, I & 140 & 180 & $290 / 260$ & $150 / 115$ & 130 & $110 / 100$ & 100 & 125 & 130 \\
\hline Royalton & $\mathrm{BP}, \mathrm{US}$ & $190 / 140$ & 180 & 260 & $150 / 130$ & 130 & 100 & $110 / 100$ & $135 / 125$ & 130 \\
\hline Ruby & $\mathrm{BP}, \mathrm{US}$ & $190 / 140$ & 180 & 260 & 120 & 130 & $110 / 100$ & $110 / 90$ & 130 & 130 \\
\hline Sam & $\mathrm{BP}, \mathrm{C}$ & 140 & $180 / 160$ & $290 / 260$ & $150 / 120$ & 130 & 100 & $110 / 100$ & $145 / 125$ & 130 \\
\hline Samba (Sumste) & $\mathrm{BP}, \mathrm{C}$ & $190 / 140$ & 180 & $290 / 260$ & $150 / 120$ & 130 & 110 & $110 / 90$ & $135 / 130$ & 130 \\
\hline Santina & $\mathrm{BP}, \mathrm{C}$ & $190 / 140$ & $180 / 160$ & $290 / 260$ & $150 / 130$ & 130 & 100 & $110 / 100$ & $135 / 125$ & 130 \\
\hline Skeena & $\mathrm{BP}, \mathrm{C}$ & 190 & 180 & $290 / 260$ & $150 / 120$ & 130 & 100 & 90 & $145 / 125$ & 130 \\
\hline Somerset & BP, US & $190 / 140$ & 180 & 260 & $150 / 120$ & 130 & $110 / 100$ & 100 & $135 / 125$ & $130 / 120$ \\
\hline Sonata (Sumleta) & $\mathrm{BP}, \mathrm{C}$ & 190 & 180 & 290 & $150 / 120$ & 130 & 100 & $100 / 90$ & $145 / 125$ & $130 / 120$ \\
\hline Spalding & BP, US & $190 / 140$ & 180 & 260 & $150 / 130$ & 130 & $110 / 100$ & $110 / 100$ & $135 / 125$ & $140 / 130$ \\
\hline Star & $\mathrm{BP}, \mathrm{C}$ & $190 / 140$ & $180 / 160$ & 260 & $150 / 120$ & 130 & $110 / 100$ & $110 / 100$ & $135 / 125$ & 130 \\
\hline Stark. Hardy Giant & BP, US & 140 & 180 & 260 & $150 / 120$ & 130 & $110 / 100$ & $100 / 90$ & $135 / 125$ & 130 \\
\hline Sue & $\mathrm{BP}, \mathrm{C}$ & 140 & $180 / 160$ & 260 & $150 / 120$ & 130 & 110 & $110 / 100$ & $135 / 125$ & $140 / 130$ \\
\hline Sumesi (12S.8.33) & $\mathrm{BP}, \mathrm{C}$ & 140 & 180 & $290 / 260$ & $150 / 120$ & 130 & $110 / 100$ & $110 / 100$ & $145 / 130$ & 130 \\
\hline Summit & $\mathrm{BP}, \mathrm{C}$ & 140 & $180 / 160$ & $290 / 260$ & $150 / 120$ & 130 & $110 / 100$ & 100 & 125 & 130 \\
\hline Sunburst & $\mathrm{BP}, \mathrm{C}$ & 140 & 180 & 290 & $150 / 120$ & 130 & $110 / 100$ & $100 / 90$ & $145 / 130$ & $130 / 120$ \\
\hline Sweetheart & $\mathrm{BP}, \mathrm{C}$ & $190 / 140$ & 180 & $290 / 260$ & $150 / 120$ & 130 & $110 / 100$ & $100 / 90$ & $135 / 125$ & $130 / 120$ \\
\hline Sylvia & $\mathrm{BP}, \mathrm{C}$ & 140 & $180 / 160$ & 290 & $150 / 120$ & 130 & $110 / 100$ & 100 & $145 / 125$ & 130 \\
\hline Taleguera Brillante & $\mathrm{U}, \mathrm{S}$ & 140 & $180 / 160$ & $290 / 260$ & $150 / 115$ & 130 & 100 & $100 / 90$ & 135 & 130 \\
\hline Tigre & $\mathrm{U}, \mathrm{F}$ & 140 & 180 & 290 & $150 / 115$ & 130 & 110 & $110 / 90$ & 120 & 130 \\
\hline Van & $\mathrm{BP}, \mathrm{C}$ & $190 / 140$ & 180 & $290 / 260$ & $150 / 120$ & 130 & 110 & $100 / 90$ & $135 / 125$ & $130 / 120$ \\
\hline Van Spur & $\mathrm{BP}, \mathrm{C}$ & $190 / 140$ & 180 & $290 / 260$ & $150 / 120$ & 130 & 110 & $100 / 90$ & $135 / 125$ & $130 / 120$ \\
\hline Vega & $\mathrm{BP}, \mathrm{C}$ & $190 / 140$ & 180 & 260 & $150 / 130$ & 130 & 110 & $100 / 90$ & $135 / 125$ & $140 / 130$ \\
\hline Vic & $\mathrm{BP}, \mathrm{C}$ & 140 & 180 & 260 & $150 / 120$ & 130 & $110 / 100$ & $100 / 90$ & $135 / 125$ & $140 / 130$ \\
\hline
\end{tabular}


Table 1 Continued

\begin{tabular}{|c|c|c|c|c|c|c|c|c|c|c|}
\hline Cultivar & Origin $*$ & Pchgms1 & Pchcms3 & Pchcms5 & UDP96-005 & DP98-409 & UPD98-021 & UPD98-022 & UPD97-402 & UPD98-412 \\
\hline Vignola (Duroni 2) & $\mathrm{U}, \mathrm{I}$ & 140 & 180 & 260 & $150 / 130$ & 130 & 110 & 100 & $140 / 125$ & 130 \\
\hline Vittoria & U, I & 140 & 180 & $290 / 260$ & $150 / 130$ & 130 & 110 & $100 / 90$ & $145 / 130$ & $130 / 120$ \\
\hline 13N.7.19 & $\mathrm{BP}, \mathrm{C}$ & 140 & 180 & $290 / 260$ & $150 / 120$ & 130 & $110 / 100$ & $100 / 90$ & $135 / 125$ & $130 / 120$ \\
\hline 13S.17.20 & $\mathrm{BP}, \mathrm{C}$ & $190 / 140$ & 180 & $290 / 260$ & $150 / 120$ & 130 & 110 & $110 / 90$ & 130 & 130 \\
\hline 13S.18.10 & $\mathrm{BP}, \mathrm{C}$ & $190 / 140$ & 180 & $290 / 260$ & $150 / 120$ & 130 & $110 / 100$ & $110 / 100$ & $135 / 125$ & 130 \\
\hline 13S.18.15 & $\mathrm{BP}, \mathrm{C}$ & $190 / 140$ & 180 & $290 / 260$ & $150 / 120$ & 130 & 110 & $110 / 100$ & $135 / 125$ & $130 / 120$ \\
\hline 13S.21.7 & $\mathrm{BP}, \mathrm{C}$ & 140 & 180 & 290 & $150 / 130$ & 130 & $110 / 100$ & 100 & $135 / 125$ & 130 \\
\hline 13S.27.17 & $\mathrm{BP}, \mathrm{C}$ & 140 & 180 & 260 & $150 / 120$ & 130 & 110 & $100 / 90$ & $135 / 125$ & $140 / 130$ \\
\hline 135.3 .13 & $\mathrm{BP}, \mathrm{C}$ & $190 / 140$ & 180 & 290 & $150 / 120$ & 130 & 100 & $110 / 100$ & $145 / 130$ & 130 \\
\hline 44W.11.8 & $\mathrm{BP}, \mathrm{C}$ & $190 / 140$ & 180 & 260 & $150 / 120$ & 130 & 110 & $110 / 100$ & $145 / 130$ & 130 \\
\hline 83703007 & $\mathrm{BP}, \mathrm{I}$ & $190 / 140$ & 180 & 290 & $150 / 120$ & 130 & 100 & 100 & $145 / 120$ & 130 \\
\hline 84703002 & $\mathrm{BP}, \mathrm{I}$ & $190 / 140$ & 180 & 290 & $150 / 120$ & 130 & $110 / 100$ & $100 / 90$ & $130 / 120$ & 130 \\
\hline 84704006 & $\mathrm{BP}, \mathrm{I}$ & 140 & 180 & 290 & $150 / 130$ & 130 & $110 / 100$ & $100 / 90$ & $130 / 120$ & 130 \\
\hline
\end{tabular}

*U = Unknown pedigree; $\mathrm{BP}=$ Breeding programme; $\mathrm{C}=$ Canada; $\mathrm{CE}=$ Central Europe; $\mathrm{F}=$ France; $\mathrm{I}=\mathrm{Italy} ; \mathrm{S}=\mathrm{Spain} ; \mathrm{US}=\mathrm{USA}$.

Table 2 Microsatellite primer pairs used for the identification of sweet cherry genotypes

\begin{tabular}{|c|c|c|c|c|c|c|}
\hline Locus & $\begin{array}{l}\text { SSR repeat structure } \\
\text { in peach }\end{array}$ & $\begin{array}{l}\text { Product size in } \\
\text { peach }(b p)\end{array}$ & $\begin{array}{c}\text { Size range in } \\
\text { sweet cherry }(b p)\end{array}$ & $\begin{array}{c}\text { No. of alleles in } \\
\text { peach }\end{array}$ & $\begin{array}{l}\text { No. of alleles in } \\
\text { sweet cherry }\end{array}$ & $H$ \\
\hline Pchgms $^{a}$ & $(\mathrm{AC})_{12}(\mathrm{AT})_{6}$ & 194 & 140-190 & 4 & 2 & 0.43 \\
\hline Pchcms $3^{\text {a }}$ & $(\mathrm{CT})_{19}$ & 220 & $160-180$ & 2 & 2 & 0.21 \\
\hline Pchcms5 $^{\text {a }}$ & $(\mathrm{CA})_{9}(\mathrm{TA})_{8}$ & 246 & $260-290$ & 3 & 2 & 0.47 \\
\hline UDP96-005 & $\begin{array}{c}(\mathrm{AC})_{16} \mathrm{TG}(\mathrm{CT})_{2} \\
\mathrm{CA}(\mathrm{CT})_{11}\end{array}$ & 155 & $120-150$ & 4 & 5 & 0.94 \\
\hline UDP98-409b & $(\mathrm{AG})_{19}$ & 129 & $130-160$ & 5 & 2 & 0.04 \\
\hline UPD98-021 ${ }^{\mathrm{c}}$ & $(\mathrm{GA})_{22}(\mathrm{CA})_{11}$ & 145 & $100-110$ & 2 & 2 & 0.52 \\
\hline UPD98-022 c & $(\mathrm{TG})_{12}(\mathrm{AG})_{24}$ & 139 & $90-110$ & 5 & 4 & 0.75 \\
\hline UPD $97-402^{\mathrm{b}}$ & $(\mathrm{AG})_{17}$ & 136 & $120-150$ & 3 & 7 & 0.81 \\
\hline UPD98-412 & $(\mathrm{AG})_{28}$ & 129 & $100-140$ & 5 & 4 & 0.25 \\
\hline
\end{tabular}

aSosinski et al (2000). ' ${ }^{\mathrm{C}}$ Cipriani et al (1999). 'Testolin et al (2000).

Table 3 Microsatellite primers that did not amplify scorable or polymorphic loci in sweet cherry genotypes

\begin{tabular}{|c|c|c|c|}
\hline Locus & Reference & SSR repeat structure in peach & Size range* $(b p)$ \\
\hline Pchcms1 & Sosinski et al (2000) & $(\mathrm{CA})_{9}(\mathrm{TA})_{14}(\mathrm{GA})_{13}$ & 130-190 \\
\hline UPD98-024 & Testolin et al (2000) & $(\mathrm{GT})_{19} \mathrm{TC}(\mathrm{TG})_{7}$ & $70-80$ \\
\hline UPD98-411 & Testolin et al (2000) & (TC) $)_{16}$ & $150-190$ \\
\hline UPD98-410 & Testolin et al (2000) & $(\mathrm{AG})_{23}$ & $130-160$ \\
\hline Pchgms2 & Sosinski et al (2000) & $(\mathrm{CT})_{24}$ & 135 n.p. \\
\hline Pchgms3 & Sosinski et al (2000) & $(\mathrm{CT})_{14}$ & 190 n.p. \\
\hline Pchgms4 & Sosinski et al (2000) & $(\mathrm{CT})_{21}$ & 150 n.p. \\
\hline Pchcms2 & Sosinski et al (2000) & $(\mathrm{CA})_{8}$ & 90 n.p. \\
\hline UDP96-003 & Cipriani et al (1999) & $(\mathrm{CT})_{11}(\mathrm{CA})_{28}$ & 85 n.p. \\
\hline UDP96-008 & Cipriani et al (1999) & $(\mathrm{CA})_{23}$ & $135-155^{1}$ n.p. \\
\hline UDP96-018 & Cipriani et al (1999) & $(\mathrm{AC})_{21}$ & 265 n.p. \\
\hline UDP96-019 & Cipriani et al (1999) & $(\mathrm{TG})_{18}(\mathrm{AG})_{7}$ & 320 n.p. \\
\hline UDP98-405 & Cipriani et al (1999) & $(\mathrm{AG})_{9}$ & 105 n.p. \\
\hline UDP98-406 & Cipriani et al (1999) & $(\mathrm{AG})_{15}$ & 395 n.p. \\
\hline UPD98-025 & Testolin et al (2000) & $(\mathrm{CA})_{19}$ & 70-95 n.p. \\
\hline UPD98-407 & Cipriani et al (1999) & $(\mathrm{GA})_{29}$ & n.a. \\
\hline UPD98-416 & Testolin et al (2000) & $(\mathrm{AG})_{18}$ & n.a. \\
\hline UDP96-001 & Cipriani et al (1999) & $(\mathrm{CA})_{17}$ & n.a. \\
\hline UDP97-403 & Cipriani et al (1999) & $(\mathrm{AG})_{22}$ & n.a. \\
\hline UPD96-010 & Cipriani et al (1999) & $(\mathrm{GT})_{21}(\mathrm{GAGT})_{4}(\mathrm{GA})_{18}$ & n.a. \\
\hline UPD96-013 & Cipriani et al (1999) & $(\mathrm{AG})_{22}(\mathrm{TG})_{8} \mathrm{TT}(\mathrm{TG})_{10}$ & n.a. \\
\hline UPD96-015 & Cipriani et al (1999) & $(\mathrm{CA})_{31}$ & n.a. \\
\hline UPD97-401 & Cipriani et al (1999) & $(\mathrm{GA})_{19}$ & n.a. \\
\hline UPD98-408 & Cipriani et al (1999) & $(\mathrm{CT})_{14}$ & n.a. \\
\hline UPD98-414 & Testolin et al (2000) & $(\mathrm{TC})_{24}$ & n.a. \\
\hline
\end{tabular}

${ }^{*}$ n.p. $=$ no polymorphism; n.a. $=$ no or weak amplification. 
ous differentiation of 72 (94\%) genotypes. UPGMA cluster analysis revealed several possible dendrograms and the dendrogram with the highest copehenetic correlation coefficient (0.66) is shown in Figure 1. As expected the genotypes derived by mutation could not be distinguished from the original genotype ('Van Spur' and 'Early Van Compact' from 'Van' and 'Burlat C1' from 'Burlat'). The other genotypes that could not be distinguished are two pairs of genotypes that share a common pedigree ('Celeste' and '13S.21.7' and 'Burlat' and '84704006'). Most genotypes obtained from the same breeding programmes and/or sharing common parents, group together.

Since most of the recently released genotypes share common parents, UPGMA cluster analysis was performed with the 23 genotypes that are not recent releases from breeding programmes. Those genotypes include the ancient cultivars of unknown origin and parental breeding genotypes directly derived from old cultivars. In this case, a single dendrogram with a cophenetic correlation coefficient of 0.70 was obtained (Figure 2). The dendrogram clustered these 23 cultivars into two main groups, although with a low bootstrap value $(<50 \%)$ support, that separate the genotypes originating from northern

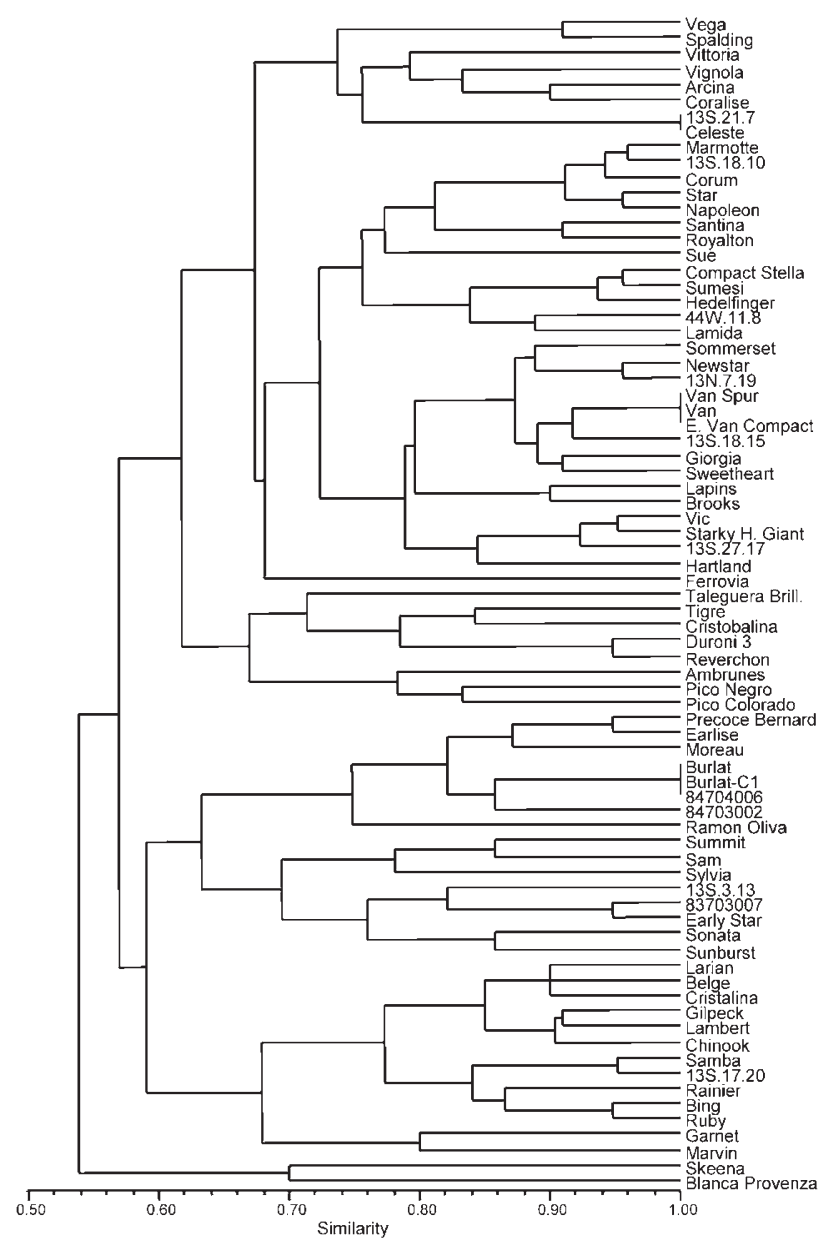

Figure 1 Dendrogram of the 76 sweet cherry cultivars included in this study based on UPGMA analysis using the similarity matrix generated by the Nei and Li coefficient after amplification with nine pairs of microsatellite primers.

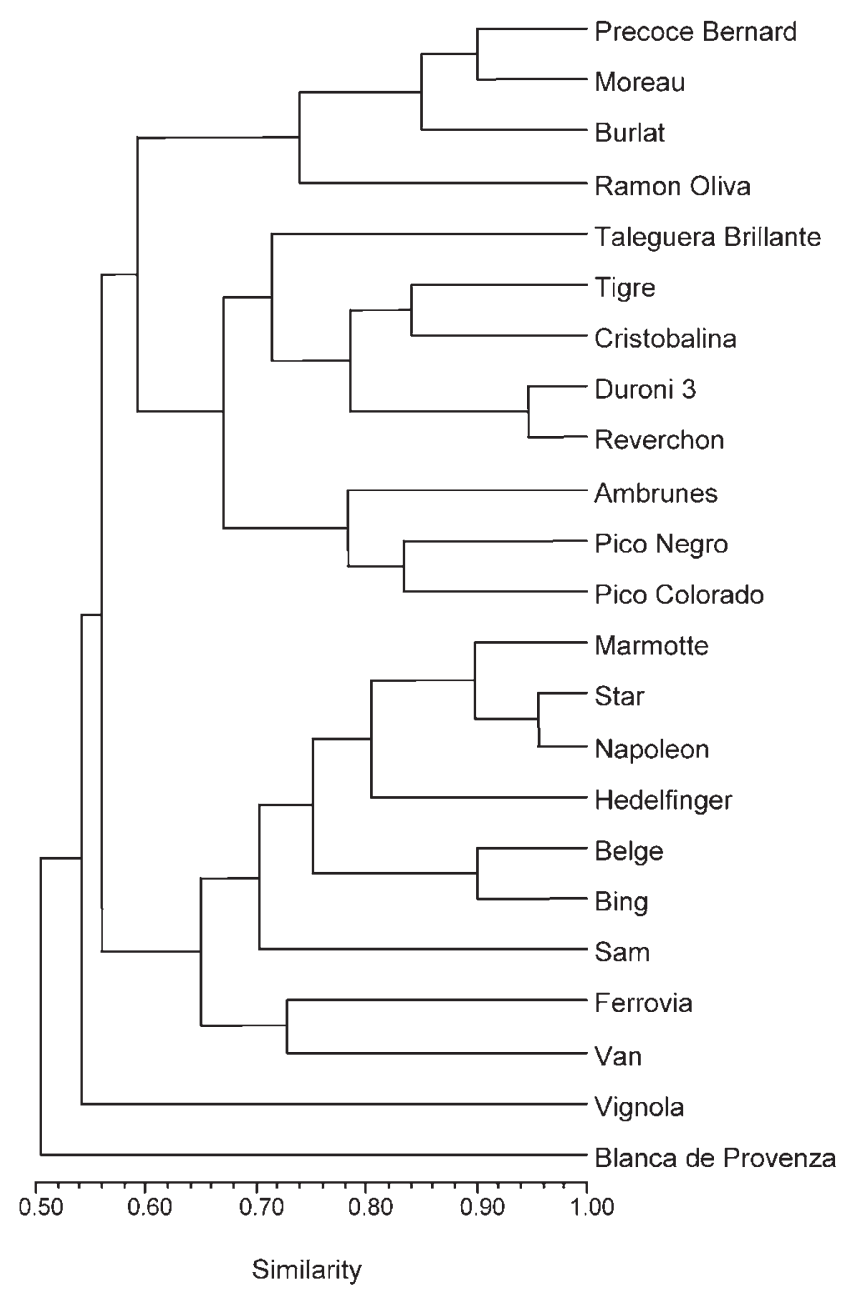

Figure 2 Dendrogram of 23 ancient or directly derived from ancient sweet cherry cultivars based on UPGMA analysis using the similarity matrix generated by the Nei and Li coefficient after amplification with nine pairs of microsatellite primers.

Europe and North America from those originating from southern Europe.

\section{Discussion}

In this work 72 sweet cherry genotypes were characterised with 27 SSR markers using nine primer pairs initially developed in peach. The results confirm the efficiency of microsatellite markers for fingerprinting purposes. Moreover, they demonstrate that microsatellites are highly conserved among peach and sweet cherry allowing the use of the same SSR primers to detect intra-specific variation in related species even though the ability to amplify loci and detect polymorphism usually decreases as the genetic distance increases (Steinkellner et al, 1997; White and Powell, 1997; Maguire et al, 2000; Roa et al, 2000).

The fact that both DNA sources used, leaves and dormant buds, produce DNA of sufficient quality for SSR amplification allows the characterisation of plant material at any time of the year. SSR detection using polyacrylamide gel electrophoresis (PAGE) complicates the identification of microsatellite alleles, mainly due to the presence of ladder-like stutter patterns, with no significant improvement in resolution compared to agarose gel 
electrophoresis. Moreover, PAGE is more time-consuming and toxic than agarose gel electrophoresis. An automated detection system would be able to resolve allelic variation at a finer scale and, consequently, the number of alleles detected would even be greater than that reported in this work. However, for routine SSR genotype analysis, agarose gel electrophoresis is a convenient method (Morgante et al, 2001).

In this work, amplification of the microsatellite loci in sweet cherry was possible for 24 of the $34(73 \%)$ peach microsatellites, of which $13(38 \%)$ were polymorphic. This efficiency is lower than that recently reported in apricot (Prunus armeniaca L.), where $88 \%$ of peach SSR primers amplified loci, while $54 \%$ of them were polymorphic (Hormaza, 2002) probably due to the different position of apricot and cherry in relation to peach in Prunus phylogeny. Thus, morphological studies (Westwood, 1993) supported by cpDNA variation (Badenes and Parfitt, 1995) classifies Prunus species into five subgenera (Prunophora, Amygdalus, Cerasus, Padus and Laurocerasus). The main cultivated Prunus species belong to three of them: Amygdalus (peach and almond), Prunophora (plum and apricot), and Cerasus (sour and sweet cherries). The studies on cpDNA variation (Badenes and Parfitt, 1995) confirm the hypothesis (Watkins, 1976) that cherries are genetically more distant from peach than apricot, since the present day subgenus Cerasus species diverged earlier from the ancestral Prunus species than the other two subgenera.

For the set of sweet cherry genotypes analysed, the mean heterozygosity of the polymorphic loci was 0.49 ranging from 0.04 to 0.94 . Although sweet cherry is an outbreeding species, the mean heterozygosity reported here is similar to that reported for other Prunus species, including peach $(0.47$, Testolin et al, 2000; 0.45, Sosinski et al, 2000) and apricot (0.51, Hormaza, 2002) using SSR markers. A low level of polymorphism in sweet cherry has also been detected by Stockinger et al (1996) and Gerlach and Stösser (1997), using RAPD markers, and by Boskovic and Tobutt (1998) and Beaver et al (1995) with isozyme markers, probably reflecting a narrow genetic base in sweet cherry germplasm.

Neither 'Burlat C1', a compact mutation of 'Burlat', nor 'Van Spur' or 'Early Van Compact', both mutations of 'Van', could be differentiated from 'Burlat' or 'Van', respectively. The impossibility of differentiating mutants that differ from the original genotype in one or few genes, was expected due to the intrinsic nature of SSRs since it is very unlikely that the microsatellites amplified correspond to the mutated DNA region when they have been randomly isolated from the whole genome. The case of the other two pairs of genotypes 'Celeste' and '13S.21.7', and '84704006' and 'Burlat' can be explained regarding the pedigree information available. 'Celeste' and '13S.21.7' both originated from the cross of 'Van' and 'Newstar', and '84704006' descends from the cross of 'Burlat' and 'Sunburst'. In the first situation, the two genotypes appear to have inherited the same alleles for the loci analysed, and, in the second, the allele segregation has resulted in the same band profile as one of the parental genotypes, 'Burlat'. The use of more SSRs recently developed in Prunus (Aranzana et al, 2002; Cantini et al, 2001) may allow discrimination between these two closely related genotypes.

Since several of the genotypes share common ancestors various similar dendrograms were possible and they were ranked according to their cophenetic correlation coefficient. The phenogram generated from the SSR data illustrates the identification of the genotypes studied and groups them according to their known pedigree (Figure 1). A better representation of the similarity relationships in this species is obtained when only the 23 genotypes that include the ancient cultivars of unknown origin and parental breeding genotypes directly derived from other old cultivars are used to construct the dendrogram (Figure 2). In this case, a single dendrogram was obtained after UPGMA analysis. Consequently, we believe that this dendrogram has a real biological significance although the scarcity of markers available results in the low cophenetic correlation coefficient and the low boostrap value support obtained. This situation will likely change when additional SSRs developed in Prunus are tested in cherry.

The dendrogram grouped the 23 ancient cultivars into two main clusters according to their geographical origin and area of cultivation, probably reflecting local selection of outstanding wild genotypes. One group contains genotypes from southern Europe, and the other contains cultivars that mainly originated in northern and central Europe or in North America but derive from ancient cultivars from northern Europe. The cultivar 'Blanca de Provenza', the only cherry tree in our collection that produces cherries with yellow-white coloured skin and flesh, appears separated from the other two clusters together with the Italian cultivar 'Vignola'. Interestingly, within the group that comprises southern European cultivars, the three cultivars that originated in the Spanish Extremadura region ('Ambrunes', 'Pico Negro' and 'Pico Colorado') cluster together. Another subgroup is formed by some French cultivars ('Precoce Bernard', 'Moreau', and 'Burlat'), that were obtained in the French region of Rhone by the end of the nineteenth and the beginning of the twentieth century (Hedrick et al, 1915; Societé Pomologique de France, 1927, 1947). The cultivar 'Ramon Oliva', that clusters closer to those three French cultivars, although it is sometimes reported to be of Spanish origin (Bargioni, 1996), seems to have been found originally near Angers in France (Hedrick et al, 1915). 'Duroni-3' and 'Reverchon', that cluster closely together, were both taken to France from Italy (Ctifl, 1990; Bargioni, 1996), while 'Taleguera Brillante', 'Cristobalina' and 'Tigre' have an unclear origin. 'Tigre' is thought to come from France as well (Societé Pomologique de France, 1927) whereas 'Cristobalina' originated in eastern Spain is morphologically different and geographically separated from the other Spanish cultivars studied; furthermore, it is selfcompatible (Hugard, 1978) and the earliest variety to flower.

The second main cluster includes the ancient cultivars from Northern and Central Europe like 'Marmotte' (Northern France), 'Napoleon' (Central Europe) and 'Hedelfinger' (Central Europe probably from Germany) and the newer genotypes originating in North America or Europe but derived from cultivars from Northern Europe: 'Star' from the open pollination of 'Deacon', 'Sam' from the open pollination of a seedling of 'Windsor' (found in Ontario, Canada), 'Bing' from a seedling of 'Republican' (probably a seedling of Napoleon) and 'Van' from the open pollination of 'Empress Eugenie' (Northern France) (Hedrick et al, 1915; Societé Pomolo- 
gique de France, 1927; Ctifl, 1990; Bargioni, 1996; Brooks and Olmo, 1952, 1997). A southern European cultivar, 'Ferrovia', from the Italian region of Bari, also clusters in this subgroup.

The possibility of using SSR primers developed in a related species to detect intra-specific variation overcomes the troublesome process of their development, making a whole battery of already isolated SSR Prunus primer sequences available for the genetic characterisation of different species in the genus. Molecular characterisation of fruit tree scions and rootstocks is essential for the interests of fruit tree breeders, germplasm collections and the commercial sector such as nurseries. Since most temperate fruit tree species are vegetatively propagated, the identification of genotypes allow a standardisable reference for the identification of any cultivar and control of its propagation, independently of any factors that may limit or influence phenotypic characterisation, such as the environment, the time of year or the age of the tree. Furthermore, the possibility of studying the genetic diversity among different cultivars and populations, will benefit cherry breeding programmes by helping to take decisions on parental genotypes for crosses, and germplasm management to maximise the conserved diversity. This is becoming increasingly important to conserve the existing variability in the wild stands of this species scattered through most European and some Asian countries, especially due to the progressive narrowing of the genetic base. This situation is evidenced by the fact that the variability of traits of potential economic interest is limited in good fruit quality sweet cherry varieties (Iezzoni et al, 1990) and that all the self-compatible cultivars currently planted or used in breeding programmes derive from a single cultivar, 'Stella'. Microsatellites seem to be appropriate to fulfil those objectives and it will be of interest to expand these kind of studies to areas where it is necessary to conserve interesting cultivars or genotypes before they disappear.

\section{Acknowledgements}

We gratefully acknowledge M Herrero for her helpful comments on this manuscript, A Abbott and R Testolin for gently providing sequences of the microsatellite primers before publication, and R Gella for valuable information on sweet cherry germplasm. AW was supported by a SIA fellowship and financial support for this work was provided by INIA (Project Grant SC00-019).

\section{References}

Aranzana MJ, Garcia-Mas J, Carbo J, Arus P (2002). Development and variability analysis of microsatellite markers in peach. Plant Breeding 121: 87-92

Badenes ML, Parfitt DE (1995). Phylogenetic relationships of cultivated Prunus species from an analysis of chloroplast DNA variation. Theor Appl Genet 90: 1035-1041.

Bargioni G (1996). Sweet Cherry Scions: characteristics of the principal commercial cultivars, breeding objectives and methods. In: Webster AD, Looney NE (eds) Cherries: Crop physiology, production and uses, Cab International: Wallingford, Oxon, UK. pp 73-112.

Beaver JA, Iezzoni AF, Ramm CW (1995). Isozyme diversity in sour, sweet and ground cherry. Theor Appl Genet 90: 847-852.

Boskovic R, Tobutt KR (1998). Inheritance and linkage relationships of isoenzymes in two interspecific cherry progenies. Euphytica 103: 273-86.
Brooks RM, Olmo HP (1952). Register of fruit $\mathcal{E}$ nut varieties. 1920-1950. University of California Press: Berkeley and Los Angeles, USA.

Brooks RM, Olmo HP (1997). Register of fruit \& nut varieties, 3rd edn. ASHS Press: Alexandria, VA, USA.

Brown SK, Iezzoni AF, Fogle HW (1996). Cherries. In: Janick J, Moore JN (eds) Fruit Breeding, Vol. I. Tree and Tropical Fruits, vol. 1, John Wiley \& Sons: New York. pp 213-255.

Callen DF, Thompson AD, Shen Y, Phillips HA, Richards RI, Mulley JC, Sutherland GR (1993). Incidence and origin of "null" alleles in the (AC) $)_{n}$ microsatellite markers. Am J Hum Genet 52: 922-927.

Cantini C, Iezzoni AF, Lamboy WF, Boritzki M, Struss D (2001). DNA fingerprinting of tetraploid cherry germplasm using simple sequence repeats. J Am Soc Hort Sci 126: 205-209.

Cipriani G, Lot G, Huang W-G, Marrazo MT, Peterlunger E, Testolin R (1999). AC/GT and AG/CT microsatellite repeats in peach [Prunus persica (L.) Batsch]: isolation, characterisation and cross-species amplification in Prunus. Theor Appl Genet 99: 65-72.

Ctifl (1990). Le Cerisier "Le Cerise De Table". Ctifl: Paris.

Downey S, Iezzoni A (2000). Polymorphic DNA markers in black cherry (Prunus serotina) are identified using sequences from sweet cherry, peach and sour cherry. J Am Soc Hort Sci 125: $76-80$

Doyle JJ, Doyle JL (1987). A rapid DNA isolation procedure for small quantities of fresh leaf tissue. Phytochem Bull 19: 11-15.

Gerlach HK, Stösser R (1997). Patterns of random amplified polymorphic DNAs for sweet cherry (Prunus avium L.) cultivar identification. Angew Bot 71: 412-418.

Gianfranceschi L, Seglias N, Tarchini R, Komjanc M, Gessler C (1998). Simple sequence repeats for the genetic analysis of apple. Theor Appl Genet 96: 1069-1076.

Granger AR, Clarke GR, Jackson JF (1993). Sweet cherry cultivar identification by leaf isozyme polymorphism. Theor Appl Genet 86: $458-464$.

Guilford P, Prakash S, Zhu JM, Rikkerink E, Gardiner S, Bassett H, Forster R (1997). Microsatellites in Malus $\times$ domestica (apple): abundance, polymorphism and cultivar identification. Theor Appl Genet 94: 249-254.

Gupta PK, Varshney RK (2000). The development and use of microsatellite markers for genetic analysis and plant breeding with emphasis in bread wheat. Euphytica 113: 163-185.

Hedrick UP, Howe GH, Taylor OM, Tubergen CB, Wellington R (1915). The Cherries of New York. Report of the New York Agricultural Experiment Station for the Year 1914 II. J.B. Lyon: Albany, NY.

Hokanson SC, Szewc-MCFadden AK, Lamboy WF, MCFerson JR (1998). Microsatellite (SSR) markers reveal genetic identities, genetic diversity and relationships in a Malus $\times$ domestica Borkh core subset collection. Theor Appl Genet 97: 671-683.

Hormaza JI (1999). Early selection in cherry combining RAPDs with embryo culture. Sci Hort 79: 121-126.

Hormaza JI (2002). Molecular characterisation and similarity relationships among apricot (Prunus armeniaca L.) genotypes using simple sequence repeats. Theor Appl Genet 104: 321-328.

Hugard J (1978). Origine du pollen et varietes pollinisatrices. Le Fruit Belge 381: 11-32.

Iezzoni A, Schmidt H, Albertini A (1990). Cherries (Prunus). In: Moore JN, Ballington JR Jr (eds) Genetic Resources of Temperate Fruit and Nut Crops, vol 1, ISHS: Wageningen, The Netherlands. pp 111-173.

Litt M, Luly JA (1989). A hypervariable microsatellite revealed by in vitro amplification of a dinucleotide repeat within the cardiac muscle actin gene. Am J Hum Gen 44: 397-401.

Maguire TL, Edwards KJ, Saenger P, Henry R (2000). Characterisation and analysis of microsatellite loci in a mangrove species, Avicennia marina (Forsk.) Vierh. (Avicenniaceae). Theor Appl Genet 101: 279-285.

Morgante M, Pfeiffer A, Jurman I, Paglia G, Olivieri AM (2001). PCR analysis of SSR polymorphisms in plant using agarose 
gels. In: Karp A, Isaac PG, Ingram DS (eds) Molecular Tools for Screening Biodiversity, Kluwer Academic Publishers, Dordrecht, The Netherlands. pp 206-207.

Nei M, Li WH (1979). Mathematical model for studying genetic variation in terms of restriction endonucleases. Proc Natl Acad Sci USA 76: 5269-5273.

Roa AC, Chavarriaga-Aaguirre P, Duque MC, Maya MM, Bonierbale MW, Iglesias C, Tohme J (2000). Cross-species amplification of cassava (Manihot esculenta) (Euphorbiaceae) microsatellites: allelic polymorphism and degree of relationship. Am J Bot 87: 1647-1655.

Société Pomologique de France (1927). Le Verger Français. Catalogue Descriptif des Fruit Adoptés par le Congrès Pomologique. Réveil du Beaujolais: Villefranche.

Société Pomologique de France (1947). Le Verger Français. Catalogue Descriptif des Fruit Adoptés par le Congrès Pomologique. B. Aranud: Lyon, Paris.

Sosinski B, Gannavarapu M, Hager LD, Beck LE, King GJ, Ryder CD, Rajapakse S, Baird WV, Ballard RE, Abbott AG (2000). Characterisation of microsatellite markers in peach [Prunus persica (L.) Batsch]. Theor Appl Genet 101: 421-428.

Steinkellner H, Lexer C, Turetschek E, Glossl J (1997). Conservation of (GA)n microsatellite loci between Quercus species. Mol Ecol 6: 1189-1194.

Stockinger EJ, Mulinix CA, Long CM, Brettin TS, Iezzoni AF (1996). A linkage map of sweet cherry based on RAPD analysis of a microspore-derived callus culture population. J Hered 87: 214-218.

Tautz D (1989). Hypervariability of simple sequences as a gen- eral source for polymorphic DNA markers. Nucleic Acids Res 17: 6463-6471.

Testolin R, Marrazo MT, Cipriani G, Quarta R, Verde I, Dettori MT, Pancaldi M, Sansavini S (2000). Microsatellite DNA in peach [Prunus persica (L.) Batsch] and its use in fingerprinting and testing the genetic origin of cultivars. Genome 43: 512-520.

Watkins R (1976). Cherry, plum, peach, apricot and almond. In: Simmonds NW (eds) Evolution of Crop Plants, Longman: London and New York. pp 242-247.

Webster AD (1996). The taxonomic classification of sweet and sour cherries and a brief history of their cultivation. In: Webster AD and Looney NE (eds) Cherries: Crop physiology, production and uses, $\mathrm{CAB}$ International: Wallingford, Oxon, UK. pp 3-24.

Westwood MN (1993). Temperate-zone pomology: physiology and culture, 3rd edn. Timber Press: Portland, Or.

White G, Powell W (1997). Cross-species amplification of SSR loci in the Meliaceae family. Mol Ecol 6: 1195-1197.

Wünsch A, Hormaza JI (in press). Cultivar identification and genetic fingerprinting of temperate fruit tree species using DNA markers. Euphytica.

Yamamoto T, Kimura T, Sawamura Y, Kotobuki K, Ban Y, Hayashi T, Matsuta N (2001). SSRs isolated from apple can identify polymorphism and genetic diversity in pear. Theor Appl Genet 102: $865-870$

Yap V, Nelson RJ (1996). WinBoot: a program for performing bootstrap analysis of binary data to determine the confidence limits of UPGMA-based dendrograms. International Rice Research Institute, Manila, Philippines. 